\title{
Presence of Porphyromonas gingivalis in gingival squamous cell carcinoma
}

\author{
Joseph Katz*, Mairelys D. Onate, Kaleb M. Pauley, Indraneel Bhattacharyya, Seunghee Cha \\ Department of Oral and Maxillofacial Diagnostic Sciences, College of Dentistry, University of Florida, Gainesville \\ FL 32610, USA
}

Periodontal disease has been recently linked to a variety of systemic conditions such as diabetes, cardiovascular disease, preterm delivery, and oral cancer. The most common bacteria associated with periodontal disease, Porphyromonas gingivalis (P. gingivalis) has not yet been studied in the malignant gingival tissues. The objective of this study was to investigate the presence of $P$. gingivalis in specimens from squamous cell carcinoma patients. We have performed immunohistochemical staining to investigate the presence of $P$. gingivalis and Streptococcus gordonii (S. gordonii), a non invasive oral bacteria, in paraffin embedded samples of gingival squamous cell carcinoma $(n=10)$ and normal gingiva $(n=5)$. Staining for $P$. gingivalis revealed the presence of the bacteria in normal gingival tissues and gingival carcinoma, with higher levels (more than $33 \%, P<0.05$ ) detected in the carcinoma samples. The staining intensity was also significantly enhanced in the malignant tissue by 2 folds $(P<0.023)$ compared to specimens stained for the non-invasive $S$. gordonii. $P$. gingivalis is abundantly present in malignant oral epithelium suggesting a potential association of the bacteria with gingival squamous cell carcinoma.

Keywords: Porphyromonas gingivalis; gingival squamous cell carcinoma; periodontits; oral cancer

International Journal of Oral Science (2011) 3: 209-215. doi: 10.4248/IJOS11075

\section{Introduction}

In recent years, periodontal disease has been linked to many systemic conditions such as cardiovascular disease, low-birth weight complications in pregnancy, diabetes, pulmonary disease and cancer. The scientific rationale behind the proposed association is the long chronic nature of inflammatory process underlying periodontitis [1-2]. Recent studies support a strong relationship between certain microorganisms and various forms of cancer. The link between viral agents such as EpsteinBarr virus (EBV) and nasopharyngeal carcinoma and Burkit's lymphoma is well documented [3]. The role of $\mathrm{HPV}$ in oral and cervical squamous cell carcinoma is

*Correspondence: Joseph Katz

Tel: 001352273 6685; Fax: 0013528460588

E-mail: jkatz@dental.ufl.edu

Received 19 February 2011; Accepted 13 September 2011 increasingly appreciated [4]. Some bacteria such as Helicobacter pylori (H. pylori) have been associated with gastric cancer [5].

More recently, a link between periodontal disease and cancer has been suggested through several studies looking both at specific types of cancers and at the overall total cancer rate and the relationship to periodontal disease [6]. Oral cancer, gingival squamous cell carcinoma in particular, has been known to mimic advanced periodontal disease in clinical appearance showing similar symptoms of swelling, bleeding, tooth mobility, deep periodontal pockets, and bone destruction [7]. Many cases of gingival squamous cell carcinoma presenting clinically similar features to inflammatory periodontal or periodontal/ endodontic lesions have been reported [8]. In some cases these lesions were probably overlapping.

Porphyromonas gingivalis ( $P$. gingivalis) is a common gram negative anaerobic oral bacteria strongly associated with periodontal disease. Although this bacterium has 
been proven to invade gingival epithelial cells in vitro, studies on presence of the bacteria at different sites of oral epithelium in vivo are very scarce [9]. P. gingivalis is known for its capacity to penetrate and invade various epithelial cells. It has a sophisticated mechanism that enables it to modify the host cell defense including alteration of some host specific genes. While inside the cell, the bacteria have been shown to affect cell cycle related molecules at different stages. Most importantly, $P$. gingivalis has been shown to prevent apoptosis of epithelial cells, an inherent protective mechanism of cells affected by cancerous processes [10-11]. It successfully invades, multiplies and survives in the cytoplasm of infected cells and spreads to adjacent cells [12]. $P$. gingivalis has also the ability to protect itself from both the humoral and cellular immune system, can suppress all three mechanisms of complement activation and is resistant to killing by oxidative stress [10]. P. gingivalis can block the apoptotic pathway in gingival epithelial cells through manipulation of the JAK/Stat pathways that controls the intrinsic mitochondrial cell death pathways and prevent programmed host cell death [13]. In addition, $P$. gingivalis may inhibit host cell death by prolonging cell survival and increasing cell proliferation [14]. It was shown to be capable to attach to various gingival carcinoma cell lines [15]. A significant association between presence of $P$. gingivalis and infection with EBV, a known virus associated with head and neck cancer, has also been reported [16]. Other bacteria such as $H$. pylori were shown to be associated with inflammation of the gastric mucosa, leading to gastritis, peptic ulcers, gastric carcinoma and gastric mucosa-assocated lymphoid tissue (MALT) lymphomas [17].

Our long term hypothesis is that the bacteria may be associated with the etiopathogenesis of squamous cell carcinoma in the gingiva. The purpose of the present study was, therefore, to investigate the presence of $P$. gingivalisin gingival squamous cell carcinoma.

\section{Materials and Methods}

\section{Tissue acquisition}

After an institutional review board (IRB approval), we randomly selected de-identified biopsy paraffin embedded blocks $(n=10)$ of squamous cell carcinoma of the gingiva archived at the Division of Oral Pathology University of Florida. These specimens had a varying degree of differentiation. Five additional blocks diagnosed as a nonneoplastic gingival tissue for comparison of staining were used. Two slides were prepared from each block and each slide contained two serial sections of $4 \mu \mathrm{m}$ thickness for staining with a primary antibody or rabbit
IgG (negative control). Control and subject slides were graded and analyzed for intensity of $P$. gingivalis staining. Since we have used archived samples in a retrospective study, information regarding age, gender, and smoking habits were not available.

\section{Antigen retrieval}

Tissues were fixed in formalin and then embedded in paraffin. The sectioned slides were deparaffinized using CitriSolv (Fisher Scientific, Pittsburgh, USA), hydrated by submersing in three separate concentrations of ethanol $(100 \%, 95 \%$, and $70 \%)$, and rinsed continuously in distilled water for $5 \mathrm{~min}$. Afterward, antigen retrieval was performed by incubating slides in a $1 \mathrm{~L}$ antigen retrieval citra plus solution (BioGenex, San Ramon, USA), according to the manufacturer's recommendation. Briefly, the slides were placed into the citrate-based antigen retrieval solution at $95{ }^{\circ} \mathrm{C}$ for $20 \mathrm{~min}$. The heated slides were cooled down to room temperature for another $20 \mathrm{~min}$, rinsed with distilled water for $5 \mathrm{~min}$, and then blocked for $30 \mathrm{~min}$ with $1.5 \%$ Normal Goat Serum (Vector Laboratories, Burlingame, USA).

\section{P. gingivalis and Streptococcus gordonii Staining}

Rabbit $P$. gingivalis polyclonal antibodies (a generous gift from Dr. Richard Lamont) were raised against formalinized cells of the type strain ATCC 33277 [18]. This antibody does not react with human cells or with other bacteria at dilutions of $1: 500$ or greater. Control antibodies raised to Streptococcus gordonii (S. gordonii) strain G9B were also utilized [19]. Tissue sections were incubated with antibodies at a 1:1000 dilution for one hour at room temperature, followed by biotin-conjugated secondary antibody for one hour at room temperature, streptavidin-peroxidase for $30 \mathrm{~min}$ at room temperature, and enzyme substrate (Vector Laboratories, Burlingame, USA). As an additional control, sections were also incubated with phosphate buffered saline (PBS) only, followed by incubation with biotin-conjugated secondary antibody, streptavidin-peroxidase, and enzyme substrate. PBS washes (three washes, 5 min each) were performed between each incubation. Sections were counterstained with methyl green and visualized by light microscopy (Olympus BH2, Center Valley, USA) according to the procedure for paraffin-embedded sections [20].

\section{Data analysis}

The sections were evaluated by two pathologists who had been calibrated by comparisons of printed images in high magnification of immunohistochemistery staining over a period of few weeks. The kappa statistic was used to assess interobserver variability based on the values 
of $>0.75$ which indicate excellent agreement beyond chance. Staining intensity was rated according to the following scale: no visible staining $=0$, faint staining $=1+$, moderate staining $=2+$, and strong staining $=3+$. Extensiveness was graded semiquantitatively as $0 \%,<10 \%$, $10 \%-25 \%, 25 \%-50 \%, 50 \%-75 \%, 75 \%-90 \%$, and $>90 \%$ of positively stained cells, per high-power field. To compare all of the available data, we assigned an overall score to each case by multiplying the intensity score by the mean percentage of cells stained (extensiveness). This method has been used and verified by others [21-22]. The overall score was used as the basis for statistical comparison for Table 1. The Wilcoxon signed ranks test for related samples was used to analyze the difference in the stain intensity between the samples stained with antibody to $P$. gingivalis and those stained with antibody to $S$. gordonii.

Table 1 Grading of $P$. gingivalis and S.gordonii immunostaining

\begin{tabular}{lcc}
\hline Diagnosis based on histology & $\begin{array}{c}\text { a Intensity for } \\
\text { P. gingivalis }\end{array}$ & $\begin{array}{c}\text { a Intensity for } \\
\text { S.gordonii }\end{array}$ \\
\hline SCC extremely well diff. & + & - \\
SCC well diff. & ++ & ++ \\
SCC mod. diff. & + & - \\
SCC mod. diff. & ++ & ++ \\
SCC mod. diff. & ++ & - \\
SCC mod. to poorly diff. & +++ & ++ \\
Papillary SCC well diff. & + & + \\
Papillary SCC well diff. & + & + \\
Papillary SCC well diff. & + & - \\
Early verrucous carcinoma & ++ & - \\
\hline
\end{tabular}

SCC: squamous cell carcinoma; diff.: differentiated; mod.: moderately. ${ }^{a}$ The intensity and extensiveness were determined per high-power field. -: no staining; +: faint staining; ++: moderate staining; +++ : strong staining.

\section{Results}

Clinical and histological features of gingival squamous cell carcinoma

Clinical gingival carcinoma demonstrates irregular granular and ulcerative lesions (black arrows), which can be easily misdiagnosed for periodontal disease (Figure 1A). Neoplastic epithelium in the section of well differentiated carcinoma is apparent, forming islands and extensions invading into the subjacent connective tissue
(Figure 1B) and visualizing discernible squamous eddies. Figure $1 \mathrm{C}$ indicates that moderately differentiated carcinoma with some of the islands of invasive epithelium displaying loss of cellular differentiation (arrows) and other areas exhibit well formed keratin pearls and squamous eddies. Poorly differentiated squamous carcinoma with normal appearing epithelium (asterisk) surrounded by islands of closely clustered, highly basophilic and pleomorphic epithelium with minimal keratinization invades into the subjacent connective tissue (Figure 1D).

\section{P. gingivalis is detected in the specimens of gingival squamous cell carcinoma}

To detect presence of $P$. gingivalis, the specimens were stained with an antibody specific for $P$. gingivalis (Figure 2). P. gingivalis staining was positive in a sample of well differentiated gingival carcinoma where prominent cytoplasmic staining is noted in the malignant epithelial cells (Figures 2A, 2B) while rabbit $\operatorname{IgG}$ controls $\mathrm{C}$ and $\mathrm{D}$ showed no staining as expected except for the edges of the specimen shown in Figure 2C.

\section{P. gingivalis invades gingival squamous cell carcinoma more significantly than S. gordonii}

In order to prove the specificity of staining for $P$. gingivalis, immunohistochemical staining for non-invasive bacteria $S$. gordonii was performed on human biopsy specimens (Figure 3). Figure 3A indicates $P$. gingivalis staining in a well-differentiated squamous cell carcinoma specimen of the gingiva exhibiting florid cytoplasmic staining of invasive malignant cells whereas $S$. gordonii staining is devoid of any staining, shown in Figure 3B. $P$. gingivalis staining demonstrates strong positive cytoplasmic reactivity again in malignant epithelial cells in a sample of moderately differentiated squamous cell carcinoma of the gingival in Figures $3 \mathrm{C}$ and $3 \mathrm{D}$ in contrast to Figure 3B exhibiting absence of reactivity with antibody specific for S. gordonii. The intensity of the sections stained with $P$. gingivalis were significantly enhanced compared to those stained with $S$. gordonii (Table 1, $P<0.023)$. When each group was averaged for intensity, the $P$. gingivalis group had a 2 -fold increase in the staining intensity compared to specimens stained for $S$. gordonii.

Normal gingival tissue exhibits significantly weak staining of P. gingivalis compared to gingival carcinoma tissues

To further examine the specificity, non-neoplastic gingival tissues were stained for $P$. gingivalis and $S$. gordonii (Figure 4). This gingival tissue was positive for P. gingivalis (Figure 4A). S. gordonii staining of gingival 
tissue from normal gingival tissue showed weak staining (Figure 4C). Negative control gingival epithelium exhibiting absence of staining with rabbit IgG (Figure 4B).
The overall staining of the carcinoma group was $33 \%$ more intense than the normal gingival control group $(P<$ 0.05 )

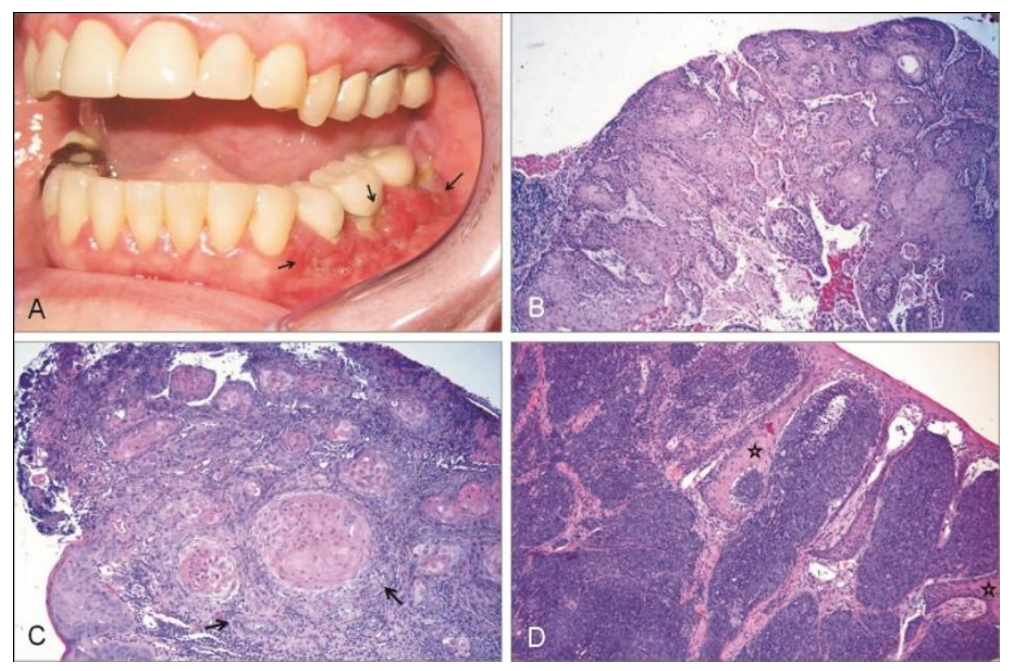

Figure 1 Clinical and histological features of gingival squamous cell carcinoma. (A) A clinical image of gingival carcinoma exhibiting irregular granular and ulcerative lesions (arrows) with recession. (B) A histology image of well differentiated squamous cell carcinoma with neoplastic epithelium forming islands and extensions that invade into the subjacent connective tissue. H\&E stain, $\times 10$. (C) An image of moderately differentiated carcinoma with some of invasive epithelium islands displaying loss of cellular differentiation (arrows) and other areas exhibiting well formed keratin pearls and squamous eddies. H\&E stain, $\times 10$. (D) Poorly differentiated squamous carcinoma with normal appearing epithelium (asterisk) surrounded by islands of closely clustered, highly basophilic and pleomorphic epithelium with minimal keratinization invading into the subjacent connective tissue. H\&E stain, $\times 10$.
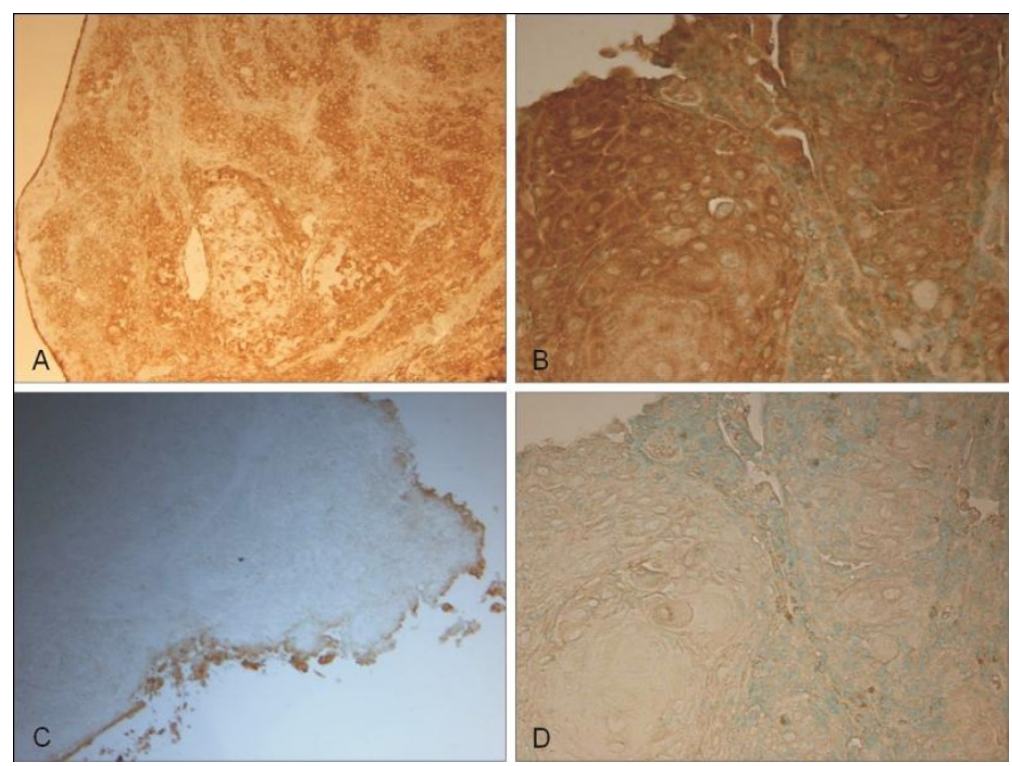

Figure 2 Detecton of $P$. gingivalis in well differentiated gingival carcinoma specimens. (A) Strongly positive staining of $P$. gingivalis in well differentiated gingival carcinoma specimens. H\&E stain, $\times 10$. (B) Strongly positive staining of $P$. gingivalis in well differentiated gingival carcinoma specimens. H\&E stain, $\times 40$. (C) Negative control exhibiting absence of staining with rabbit IgG. H\&E stain, $\times 10$. (D) Negative control exhibiting absence of staining with rabbit IgG. H\&E stain, $\times 40$. 

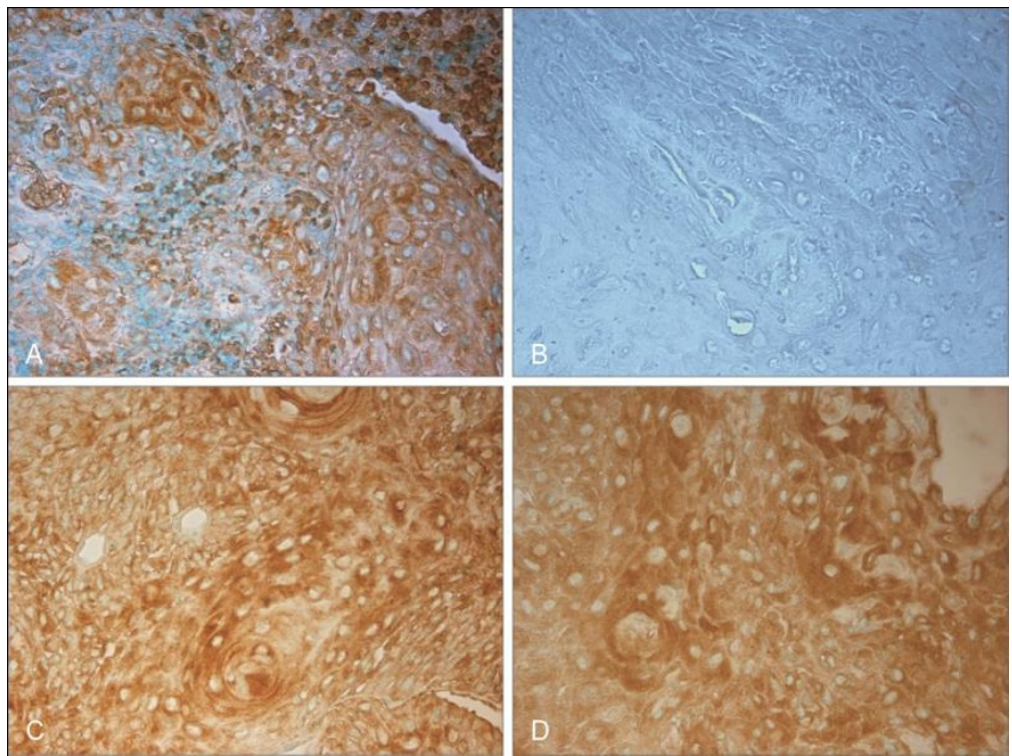

Figure 3 Strong staining of $P$. gingivalis in gingival carcinoma specimens in comparison with $S$. gordonii staining. (A) P. gingivalis staining demonstrates strong positive cytoplasmic reactivity in malignant epithelial cells in a sample of well differentiated squamous cell carcinoma of the gingival. H\&E stain, $\times 40$. (B) An image exhibits absence of reactivity with antibody specific for $S$. gordonii. H\&E stain, $\times 40$. (C) Photomicrograph of $P$. gingivalis antibody staining exhibits florid cytoplasmic staining of invasive malignant cells in a specimen of moderately differentiated gingival squamous cell carcinoma. H\&E stain, $\times 40$. (D) Photomicrograph of $P$. gingivalis antibody staining exhibits florid cytoplasmic staining of invasive malignant cells in a specimen of moderately differentiated gingival squamous cell carcinoma. H\&E stain, $\times 40$.
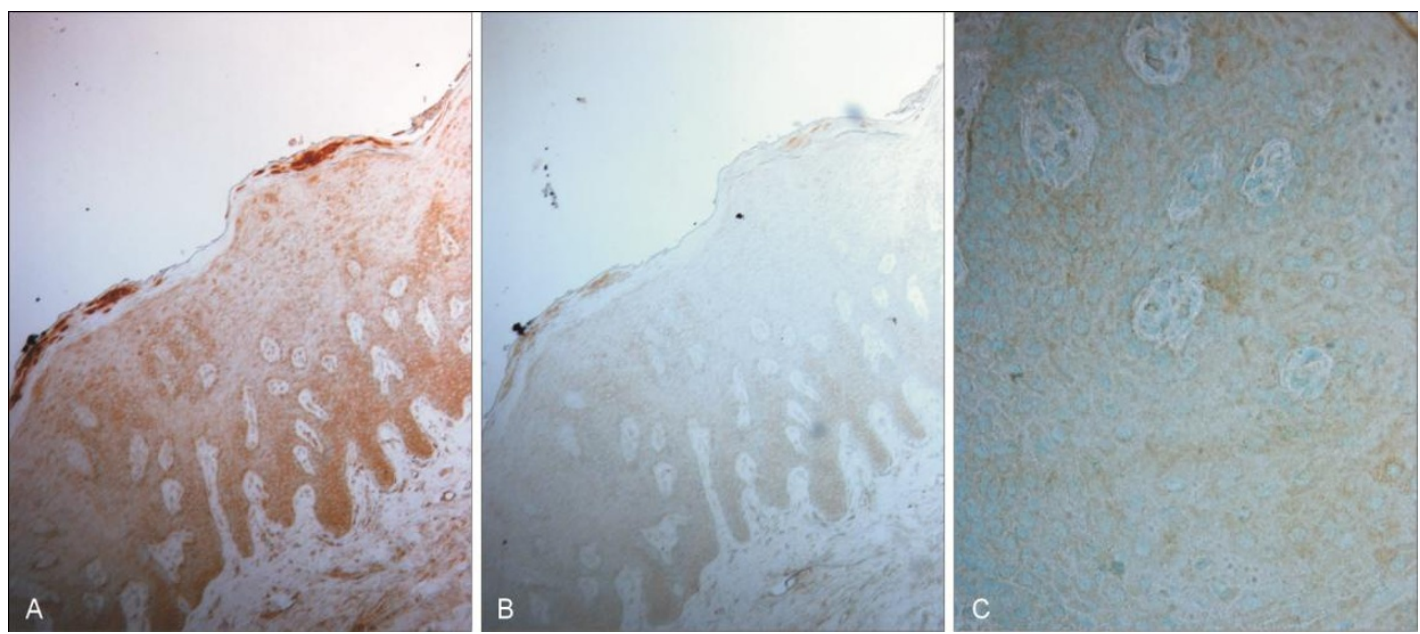

Figure 4 Normal gingival tissue staining for $P$. gingivalis. (A) Demonstrates a section of hyperplastic non-neoplastic gingival epithelium exhibiting positive staining with $P$. gingivalis antibody. H\&E stain, $\times 10$. (B) Negative control with same section of gingival hyperkeratosis demonstrating absence of staining with rabbit serum H\&E stain, $\times 10$. (C) Normal gingival tissue exhibiting weak staining with S. gordonii. H\&E stain, $\times 20$.

\section{Discussion}

In the current study, we have identified significant staining specific for $P$. gingivalis in gingival carcinoma specimens, and to a lesser extent in the normal gingival tissue. Staining was mainly localized in malignant epithelial cells in most cases. This finding indicates that at histological site, the bacteria have the capacity to 
invade both normal and neoplastic cells in vivo. It is not clear if $P$. gingivalis invaded the malignant cells before or after their transformation. We noticed that poorlydifferentiated gingival carcinoma specimens tend to show stronger $P$. gingivalis staining compared to welldifferentiated tissues. Whether there is any reliable association between the degree of differentiation and $P$. gingivalis involvement still needs to be further investigated with a larger sample size. In order to examine specificity of $P$. gingivalis staining, we further stained the specimens for $S$. gordonii. The latter is known to be a non-invasive component of the oral flora. As illustrated in Figure 3, the $P$. gingivalis staining was stronger in gingival carcinoma compared to specimens stained for $S$. gordonii. According to our statistical analyses, the intensity of the section stained with $P$. gingivalis was significantly enhanced by 2 -fold compared to $S$. gordonii stained sections. In the past, we have demonstrated the presence of $P$. gingivalis in the trophoblasts of placenta from both normal and chorioamnioitis. Interestingly, the bacteria invaded the normal trophoblast cells as well, although it was to a lesser extent [23], similar to the invasion of the normal gingival and malignant oral epithelium presented here.

Connections between oral cancer and tooth loss or periodontal disease have been evaluated in several studies [24-26]. Most found a significant increase of oral cancer risk in patients with increased tooth loss or other parameters of periodontal disease even after adjustment for tobacco and alcohol. Perhaps the most informative studies of these are two by Tezal et al [25-27], since objective measures of periodontal disease were used in these as opposed to tooth loss as a measurement tool.

The significance of our finding of increased $P$. gingivalis presence in the gingival carcinoma tissues compared to normal gingiva is not clear. Nonetheless, it is evident that $P$. gingivalis penetrates both normal and malignant epithelium at a larger extent than non-invading bacteria such as $S$. gordonii. Although there is a possibility that $P$. gingivalis may be involved in tumorigenesis by inhibiting host cell death and inducing cell proliferation, it may be presumptuous to conclude its carcinogenic potential in gingival tissue without further direct evidence.

In this retrospective study of archived samples no information is provided on co morbidity factors such as age, gender, smoking and alcohol consumption. These variables may be important as they may affect the ability of the bacteria to invade the gingival tissue and potentially impact the malignant process. Further studies using $P$. gingivalis DNA probes in carcinoma tissues at different disease stage in a large cohort is warranted to determine its significance in gingival squamous cell carcinoma etiopathology.

\section{Acknowledgments}

This work supported by Kaleb M. Pauley, is supported by National Institute of Health/National Institute of Dental and Craniofacial Research training grant T32DE007200.

\section{References}

1 Hayashi C, Gudino CV, Gibson FC, Genco CA. Review: pathogen-induced inflammation at sites distant from oral infection: bacterial persistence and induction of cell-specific innate immune inflammatory pathways. Mol Oral Microbiol 2010; 25: 305-316.

2 Michaud DS, Liu Y, Meyer M, Giovannucci E, Joshipura K. Periodontal disease, tooth loss, and cancer risk in male health professionals: a prospective cohort study. Lancet Oncol 2008; 9: 550-558.

3 Kutok JL, Wang F. Spectrum of Epstein-Barr virus-associated diseases. Annu Rev Path 2006; 1: 375-404.

4 Mork J, Lie AK, Glattre E, et al. Human papillomavirus infection as a risk factor for squamous-cell carcinoma of the head and neck. N Engl J Med 2001; 344: 1125-1131.

5 Polk DB, Peek RM. Helicobacter pylori: gastric cancer and beyond. Nat Rev Cancer 2010; 10: 403-414.

6 Fitzpatrick SG, Katz J. The association between periodontal disease and cancer: a review of the literature. J Dent 2010; 38: 83-95.

7 Seoane J, Varela-Centelles PI, Walsh TF, Lopez-Cedrun JL, Vazquez I. Gingival squamous cell carcinoma: diagnostic delay or rapid invasion? J Periodontol 2006, 77: 1229-1233.

8 Yoon TY, Bhattacharyya I, KatzJ, Towle HJ, Islam MN Squamous cell carcinoma of the gingiva presenting as localized periodontal disease. Quintessence Int 2007; 38: 97-102.

9 Kim YC, Ko Y, Hong SD, et al. Presence of Porphyromonas gingivalis and plasma cell dominance in gingival tissues with periodontitis. Oral Dis 2010; 16: 375-381.

10 Hajishengallis G. Porphyromonas gingivalis-host interactions: open war or intelligent guerilla tactics? Microbes Infect 2009; 11: 637-645.

11 Yilmaz O. The chronicles of Porphyromonas gingivalis: the microbium, the human oral epithelium and their interplay. Microbiology 2008; 154: 2897-2903.

12 Yilmaz O, Verbeke P, Lamont RJ, Ojcius DM. Intercellular spreading of Porphyromonas gingivalis infection in primary gingival epithelial cells. Infect Immun 2006; 74: 703-710.

13 Mao S, Park Y, Hasegawa Y, et al. Intrinsic apoptotic pathways of gingival epithelial cells modulated by Porphyromonas gingivalis. Cell Microbio 2007; 9: 1997-2007.

14 Kuboniwa M, Hasegawa Y, Mao S, et al. P. gingivalis 
accelerates gingival epithelial cell progression through the cell cycle. Microbes Infect 2008; 10: 122-128.

15 Hirose K, Isogai E, Mizugai H, Ueda I. Adhesion of Porphyromonas gingivalis fimbriae to human gingival cell line Ca9-22. Oral Microbiol Immunol 1996; 11: 402-406.

16 Slots J, Kamma JJ, Sugar C. The herpesvirus-Porphyromonas gingivalis-periodontitis axis. J Periodontal Res 2003; 38: 318-323.

17 Tsai HF, Hsu PN. Interplay between Helicobacter pylori and immune cells in immune pathogenesis of gastric inflammation and mucosal pathology. Cell Mol Immunol 2010; 7: 255259 .

18 Yilmaz O, Watanabe R, Lamont RJ. Involvement of integrins in fimbriae-mediated binding and invasion by Porphyromonas gingivalis. Cell Microbiol 2002; 4: 305-314.

19 Rosan B, Eifert R, Baker CT, Lamont RJ. Isolation and characterization of a non-adherent mutant of Streptococcus sanguis G9B. Oral Microbiol Immunol 1998; 3: 153-161.

20 Koenig JM, Chegini N. Enhanced expression of Fasassociated proteins in decidual and trophoblastic tissues in pregnancy-induced hypertension. Am J Reprod Immunol 2000; 44: 347-349.
21 Patel M, Lu L, Zander DS, et al. ALDH1A1 and ALDH3A1 expression in lung cancers: correlation with histologic type and potential precursors. Lung Cancer 2008; 59: 340-349.

22 Luo J, Zha S, Gage WR, et al. Alpha-methylacyl-CoA racemase: a new molecular marker for prostate cancer. Cancer Res 2002; 62: 2220-2226.

23 Katz J, Chegini N, Shiverick KT, Lamont RJ. Localization of $P$. gingivalis in preterm delivery placenta. $J$ Dent Res 2009; 88: 575-578.

24 Bundgaard T, Wildt J, Frydenberg M, Elbrond O, Nielsen JE. Case-control study of squamous cell cancer of the oral cavity in Denmark. Cancer Causes Contro 1995; 6: 57-67.

25 Hiraki A, Matsuo K, Suzuki T, Kawase T, Tajima K. Teeth loss and risk of cancer at 14 common sites in Japanese. Cancer Epidemiol Biomarkers Prev 2008; 17: 1222-1227.

26 Tezal M, Sullivan MA, Hyland A, et al. Chronic periodontitis and the incidence of head and neck squamous cell carcinoma. Cancer Epidemiol Biomarkers Prev 2009; 18: 2406-2412.

27 Tezal M, Sullivan MA, Reid ME, et al. Chronic periodontitis and the risk of tongue cancer. Arch Otolaryngol Head Neck Surg 2007; 133: 450-454. 